\title{
Neurobiology of behavior: exploring the frontier between the mind and the brain
}

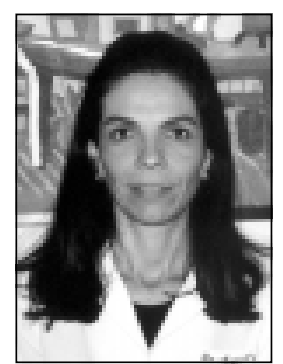

Magda L. Nunes ${ }^{1}$

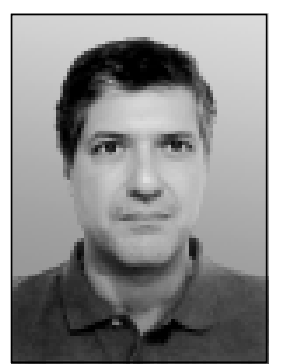

Marcos T. Mercadante 2
The discussion of psychological and biological issues used to be a considerable epistemological exercise two decades ago; today, this discussion can be held in pragmatic terms, allowing a certain topic to be analyzed under a more comprehensive and advantageous theoretical principle. This possibility has become a reality thanks to the improvement and development of new neuroimaging techniques, and genetic and molecular biology studies.

Nowadays, technological advance allows us to develop animal models that resemble real pathological situations, helping us to elucidate these pathologies, and opening new therapeutic perspectives. A recent study showed that mice knocked-out for the serotonin receptor gene are more anxious than their peers in which this gene is expressed. As, in this experiment, this gene could be expressed at any time during development, it was made clear that to have an anxious (insecure?) animal, the gene had to be silent during the first fifteen days of life. After this period, whether or not the gene was active was no longer important. ${ }^{1} \mathrm{~A}$ fascinating and intriguing finding is that in nature it is maternal care that induces the expression of this gene, that is, if mother mouse does not lick its pups,

1. PhD. Professor, School of Medicine, Pontifícia Universidade Católica do Rio Grande do Sul (PUCRS), Porto Alegre, RS, Brazil.

2. PhD. Associate professor, Postgraduate Program in Development Disorders, Universidade P. Mackenzie, São Paulo, SP, Brazil. they do not express this gene from the DNA strand. Psychology had already mentioned the importance of the mother/baby interaction more than one century ago, now this is confirmed by biology. This undoubtedly shows that our options have been expanded.

Other resources (e.g.: models that use technologies such as eye tracking) allow us to decode what is explored by an individual in a given scene. Interestingly enough, individuals with pervasive developmental disorders (e.g.: autistic persons) tend to look at the mouth region when they observe a human face, differently from healthy controls who tend to look at the eye region. ${ }^{2}$ Once again, the biological proposal matches psychological knowledge. If the importance of a child looking at his/her mother has been known for years, now we can determine to what extent the inability to explore this region affects the development of social skills. Since autistic children do not establish eye contact, they cannot "follow" emotional and communicative variations that result from the interaction between two people. Now, thanks to neuroimaging studies, we are close to admitting that changes in the circuit comprised by the cerebellum, corpus striatum, and cerebral cortex could be the initial step towards the elucidation of these disorders. ${ }^{3}$

Thus, if on the one hand we are relatively acquainted with the knowledge provided by psychology, and most professionals that treat children and adolescents in Brazil master this language; on the other hand, we have to 
explore new grounds. With rich and varied instruments at hand, we will have more resources to treat our patients.

It was a challenge to come up with a supplemental issue of Jornal de Pediatria that could present a psychological and biological view of the most frequent behavioral disorders observed in childhood and adolescence and the major neuropsychiatric symptoms associated with them. This marks a further milestone in the evolution of our journal.

The current supplement includes 13 articles, which offer an update on the latest findings about behavioral neurobiology. The knowledge of psychological and biological aspects of behavioral disorders will allow pediatricians to improve their clinical assessment of these patients.
Nevertheless, this is just the beginning, since there is a large volume of information on neuroscience being produced at research centers. Much more is yet to come!

\section{References}

1. Gross C, Zhuang X, Stark K, Ramboz S, Oosting R, Kirby L, et al. Serotonin $1 \mathrm{~A}$ receptor acts during development to establish normal anxiety-like behaviour in the adult. Nature. 2002;416:396-400.

2. Klin A, Jones W, Schultz R, Volkmar F, Cohen D. Defining and quantifying the social phenotype in autism. Am J Psychiatry. 2002;159:895-908.

3. Dawson G, Webb S, Schellenberg GD, Dager S, Friedman S, Aylward $E$, et al. Defining the broader phenotype of autism: genetic, brain, and behavioral perspectives. Dev Psychopathol. 2002; $14: 581-611$ 